\title{
Reformstau in der Ausbildung von Ärztinnen und Ärzten?
}

Die Fachgesellschaften der Grundversorgung machen sich grosse Sorgen wegen Aktivitäten um die Aus- und Weiterbildung der Ärztinnen und Ärzte, welche den seit einigen Jahren laufenden Reformprozess zum Stocken bringen. Sie wenden sich in einem Brief, der in dieser Ausgabe der SÄZ auf Seite 1729 veröffentlicht wird, an die Interfakultätskommission, eine Arbeitsgemeinschaft aller an der Ausbildung Involvierten, welche stolz auf die Einführung eines gemeinsamen Lernzielkataloges an allen fünf medizinischen Fakultäten blicken darf. Erste Resultate dieses grossen Reformprozesses sind in gut fünf Jahren zu erwarten. Dieser Prozess soll nun zielgerichtet vorangetrieben und nicht wie so vieles im Bildungswesen auf der Strecke unter dem Motto einer «Bildungsreform» geändert, sprich abgebrochen, werden. Die vertikale Ausrichtung der Ausbildung mit der Möglichkeit zur
Wahl von Kern- und Mantelfächern sollte nicht dem Bologna-Prozess geopfert werden.

Nachdem mit viel Energie an einem Medizinalberufegesetz gearbeitet wurde, scheint dessen Entwurf in den Schubladen der Verwaltung zu verschwinden. Zwar werden Begriffe wie «Kontinuum von Ausbildung und Weiterbildung» weiterhin verwendet; Begriffe wie «Systemsteuerung» und «Finanzierungsmechanismen» jedoch gewinnen an Bedeutung. Das Ziel der Reform hat sich hoffentlich nicht geändert: Eine effiziente auf die Bedürfnisse der Gesellschaft, namentlich der Patientinnen und Patienten, ausgerichtete Aus- und Weiterbildung von Ärztinnen und Ärzten mit entsprechenden fachlichen, sozialen, kommunikativen und menschlichen Kompetenzen.

Max Giger, Mitglied Zentralvorstand FMH

\section{Blocage dans la réforme de la formation médicale?}

Les sociétés de médecine de premier recours sont fortement préoccupées par les diverses initiatives visant actuellement à modifier la formation médicale prégraduée et postgraduée, initiatives qui risquent d'entraver la poursuite du processus de réforme engagé depuis plusieurs années déjà. Ces sociétés se sont adressées par une lettre, que nous publions dans ce numéro du BMS à la page 1730, à la Commission interfacultés médicale suisse, communauté de travail regroupant toutes les instances impliquées dans la formation, qui peut se flatter d'avoir réussi à introduire un catalogue d'objectifs d'apprentissage commun aux cinq facultés de médecine. Les premiers résultats de cet important processus de réforme seront tangibles dans un peu plus de cinq ans. Ce processus doit donc être poursuivi de manière ciblée et non pas, comme cela se fait souvent dans le domaine de la formation, être modifié, voire interrompu en cours de route sous le couvert d'une «réforme de la formation». L'orientation verticale de la formation avec des possibilités de choix parmi des branches princi- pales et à option ne devrait pas être sacrifiée sur l'autel des directives de Bologne.

On constate maintenant qu'après tous les efforts déployés pour l'élaboration d'une nouvelle loi sur les professions médicales, le projet semble dormir dans les tiroirs de l'administration fédérale. Et s'il est vrai que la notion de «continuum» est toujours appliquée à la formation prégraduée et postgraduée, on n'en relève pas moins que des notions telles que «pilotage du système» et «mécanismes de financement» sont en train de gagner en importance. Mais le but de la réforme, espérons-le, demeure inchangé, à savoir: une formation médicale prégraduée et postgraduée efficace, axée sur les besoins de la société, notamment ceux des patientes et des patients et permettant aux médecins en formation d'acquérir des compétences professionnelles, sociales, communicatives et humaines.

Max Giger, membre du Comité central FMH 